tients have a titer of 1:160 at one week. However, in an outbreak involving type A organisms, that titer would be considered diagnostic of tularemia, because few serologic surveys have been performed in areas where infection with the type A strain is endemic and in persons who have no history of tularemia. Patients who have had type A pneumonitis do have persistent agglutinating-antibody titers for several years and should be immune to reinfection, if they are immunocompetent.

Pérez-Castrillón et al. have reported the use of fluoroquinolones in the treatment of patients with tularemia. I was aware of their report and should have acknowledged their work. In their study, the less virulent form of $F$. tularensis was the causative microbe. Presumably, disease caused by type A organisms will be as responsive as the less virulent form. However, only a few patients with type A disease have been successfully treated.

Richard Hornick, M.D.

Orlando Regional Healthcare

Orlando, FL 32806

dickh@orhs.org

1. Tularemia - Oklahoma, 2000. MMWR Morb Mortal Wkly Rep 2001; 50:704-6.

\section{Case 38-2001: Paraneoplastic Encephalomyelitis and Sensory Ganglioneuropathy}

To the Editor: In discussing the case of paraneoplastic ganglionopathy with encephalopathy (Dec. 13 issue) ${ }_{1}^{1}$ Amato and Anderson focused on the differential diagnosis and the causes of sensory ganglionopathies. Because of the primary impairment of T-shaped sensory neurons, ganglionopathies are characterized by a specific pattern of joint, muscle, and skin denervation involving myelinated and unmyelinated axons in a fashion that is not length-dependent, leading to distinct clinical, neurophysiological, and neuropathological findings. Typically, skin biopsy demonstrates similar decreases in fiber density at distal and proximal sites. ${ }^{2}$ Moreover, evidence of involvement of the central sensory pathway can be used to localize the disease process to the dorsal-root ganglia and differentiate ganglionopathies from axonal neuropathies. Magnetic resonance imaging (MRI) of the spinal cord can show high signal intensity on $\mathrm{T}_{2}$-weighted images throughout the length of the posterior columns. ${ }^{3}$ In this case, the linear hyperintensity on the posterior aspect of the cervical spine probably reflected the degeneration of central sensory projections observed on neuropathological examination and would have been better demonstrated by axial MRI scanning at different levels.

The authors report a number of disorders associated with sensory ganglionopathies, but additional disorders with such associations include chronic autoimmune hepatitis, ${ }^{3}$ among autoimmune diseases, and infection with Epstein-Barr virus ${ }^{4}$ varicella-zoster virus, ${ }^{5}$ or measles virus, among infectious diseases. Finally, sensory neurons of dorsal-root ganglia are primarily affected in many genetic diseases, including hereditary sensory autonomic neuropathies, Fabry's disease, vi- tamin E deficiency, Friedreich's ataxia, and other types of spinocerebellar degeneration. . $^{3,6}$

\author{
GiUseppe LaURia, M.D. \\ Davide Pareyson, M.D. \\ Angelo Sghirlanzoni, M.D. \\ National Neurological Institute C. Besta \\ 20133 Milan, Italy \\ g_lauria@libero.it
}

1. Case Records of the Massachusetts General Hospital (Case 38-2001). N Engl J Med 2001;345:1758-65.

2. Lauria G, Sghirlanzoni A, Lombardi R, Pareyson D. Epidermal nerve fiber density in sensory ganglionopathies: clinical and neurophysiologic correlations. Muscle Nerve 2001:24:1034-9.

3. Lauria G, Pareyson D, Grisoli M, Sghirlanzoni A. Clinical and magnetic resonance imaging findings in chronic sensory ganglionopathies. Ann Neurol 2000;47:104-9.

4. Rubin DI, Daube JR. Subacute sensory neuropathy associated with Epstein-Barr virus. Muscle Nerve 1999;22:1607-10.

5. Ramos F, Monforte C, Luengo A. Acute sensory neuropathy associated with a varicella-zoster infection. Rev Neurol 1999;28:1067-9. (In Spanish.) 6. Thomas PK. Hereditary sensory neuropathies. Brain Pathol 1993;3:157-63.

\section{Dr. Amato replies:}

To the Editor: Lauria and colleagues have performed elegant studies of epidermal nerve fiber density ${ }^{1}$ and spinal MRI findings ${ }^{2}$ in patients with sensory ganglionopathies. With regard to the case of paraneoplastic encephalomyelitis and sensory ganglioneuropathy, they suggest that the linear abnormality noted on the MRI scan of the cervical spine may be secondary to degeneration of the posterior columns related to the sensory ganglionopathy. However, I chose not to overinterpret this subtle abnormality, which was present only on the sagittal image, because concurrent signal changes were not noted on the axial images. Skin-biopsy specimens were not readily available, and more important, would not have provided any more information that would have been useful for localization than was already available from the clinical history and examination.

The time constraints of the clinicopathological conference did not allow me to present a differential diagnosis of every reported disease associated with damage to the sensory ganglia. Instead, I tried to review the pertinent aspects of the patient's history, neurologic examination, and workup that would allow for a reasonable differential diagnosis and specification of further diagnostic testing required to make the correct diagnosis. In a patient with known small-cell lung cancer, an altered mental status, pseudoathetosis, myoclonus, and sensory ataxia, there was no differential diagnosis. Nevertheless, I tried to discuss common neuropathies that occur in the setting of cancer. I would never consider chronic autoimmune hepatitis infection with Epstein-Barr virus, varicella-zoster virus, or measles virus or any of the inherited neuropathies listed by Lauria and colleagues as possible causes of this patient's neurologic deterioration. The patient had an obvious paraneoplastic disorder, which was eventually confirmed by the demonstration of anti-Hu antibodies in the serum and cerebrospinal fluid and by the autopsy findings.

Anthony A. Amato, M.D. Brigham and Women's Hospital Boston, MA 02115 aamato@partners.org 